\title{
Progressive Multifocal Leukoencephalopathy -A Case Report in an Immunocompetent Patient
}

\author{
Ana Faceira ${ }^{1,2^{*}}$, Daniela Magalhães ${ }^{2,3}$, Filipa Ceia ${ }^{2,4}$, João Nuak ${ }^{2,5}$, Carina Reis ${ }^{6}$, Susana Ferreira ${ }^{1}$ \\ ${ }^{1}$ Internal Medicine Department, Hospital de São João, Porto, Portugal \\ ${ }^{2}$ Faculdade de Medicina da Universidade do Porto, Porto, Portugal \\ ${ }^{3}$ Endocrinology, Diabetes and Metabolism Department, Hospital de São João, Porto, Portugal \\ ${ }^{4}$ Instituto de Investigação e Inovação em Saúde, Universidade do Porto, Porto, Portugal \\ ${ }^{5}$ Infectious Diseases Department, Hospital de São João, Porto, Portugal \\ ${ }^{6}$ Neuroradiology Department, Hospital de São João, Porto, Portugal \\ Email: ${ }^{\star}$ faceira.ana@gmail.com
}

How to cite this paper: Faceira, A., Magalhães, D., Ceia, F., Nuak, J., Reis, C. and Ferreira, S. (2018) Progressive Multifocal Leukoencephalopathy-A Case Report in an Immunocompetent Patient. Advances in Infectious Diseases, 8, 10-16. https://doi.org/10.4236/aid.2018.81002

Received: December 17, 2017

Accepted: January 23, 2018

Published: January 26, 2018

Copyright $\odot 2018$ by authors and Scientific Research Publishing Inc. This work is licensed under the Creative Commons Attribution International License (CC BY 4.0).

http://creativecommons.org/licenses/by/4.0/

\begin{abstract}
Progressive multifocal leukoencephalopathy (PML) is a demyelinating disease of the central nervous system due to the reactivation of the JC virus, which usually occurs in immunocompromised patients and is a major opportunistic infection associated with HIV infection. We report a case of a previously healthy patient who was diagnosed with PML.
\end{abstract}

\section{Keywords}

Immunosuppression, Immunocompetent, JC Virus, Progressive Multifocal Leukoencephalopathy

\section{Introduction}

JC virus (JVC) is a double-stranded circular DNA virus, which belongs to the Polyomaviridae family and to the Polyomavirus genus. The polyomaviruses are ubiquitous in nature and are species-specific, similar to the JCV in humans. It is assumed that the major routes of the virus's transmission are inhalation and ingestion of contaminated water [1] [2].

JCV is the etiologic agent of a demyelinating disease of the central nervous system (CNS) - progressive multifocal leukoencephalopathy (PML). The pathogenesis of PML is divided into 3 phases. The primary infection is usually asymptomatic, with approximately $85 \%$ of the adult population showing antibod- 
ies against the JCV, and thereafter the virus remains as a persistent latent infection in the urinary tract, bone marrow and spleen. The final phase is related to the reactivation and dissemination of the virus with an almost certain hematogenous spread to the CNS [1] [2] [3].

\section{Case Report}

A 68-year-old Caucasian male was evaluated in a medical consultation with a history of dysmetria of the right limbs, dysdiadochokinesia, limb ataxia and dysarthria, which lasted for approximately one month. The brain magnetic resonance imaging (MRI) showed a T2 hyperintense (Figure 1, Figure 2) and T1 hypointense lesion in the right cerebellar hemisphere and middle cerebellar peduncle, with no mass effect or gadolinium enhancement (Figure 3), and diffusion-weighted imaging (DWI) showed predominantly facilitated diffusion of water molecules, with patchy peripheral high signal but with no apparent diffusion coefficient (ADC) lowering-no restriction (Figure 4, Figure 5). An extensive complementary study was carried out, including: cerebrospinal fluid (CSF) analysis (microbiological, immunophenotypic and cytological studies with malignant cell research), immunological study (antineutrophil cytoplasmic antibodies, antinuclear antibodies, anti-double stranded DNA antibodies) and investigation to exclude a solid neoplasia/lymphoma (upper gastrointestinal endoscopy, colonoscopy, thoraco-abdominopelvic computed tomography scan, serum protein electrophoresis) or an infectious disease (particularly HIV infection). This study was inconclusive and did not allow an accurate diagnosis. Thus, because it was not possible to exclude that it was a subacute cerebral infarction, antiplatelet therapy with clopidogrel and motor rehabilitation treatment were set.

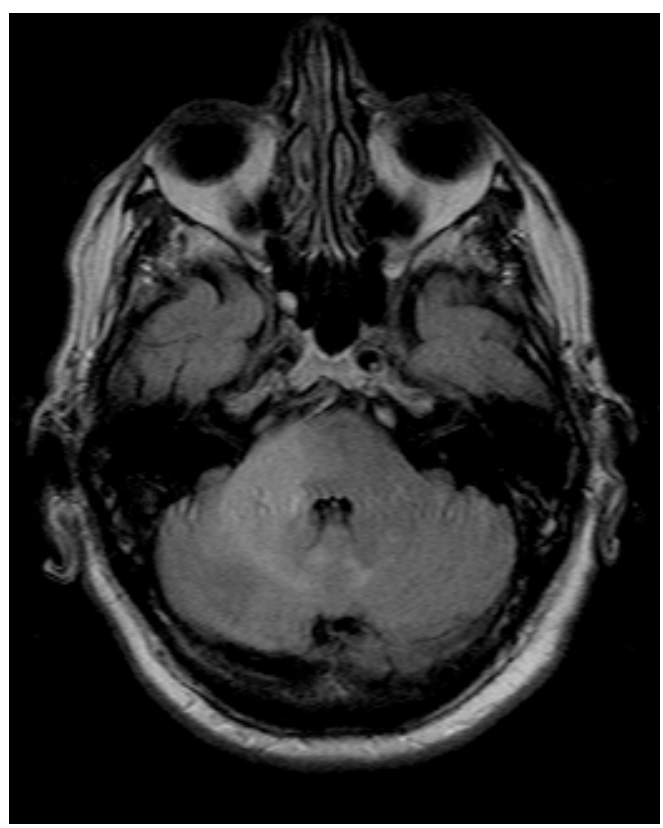

Figure 1. Axial T2 FLAIR. 


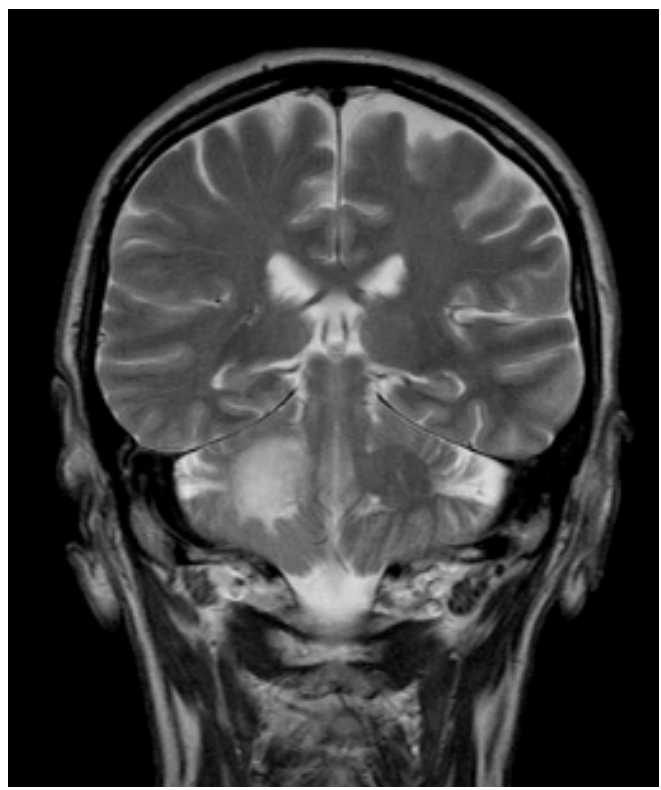

Figure 2. Coronal T2 TSE.

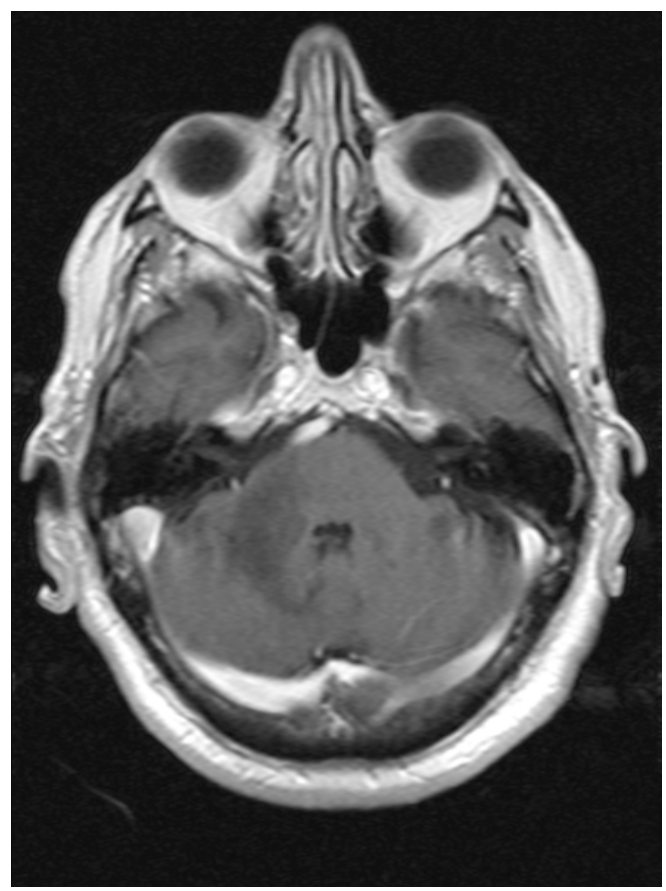

Figure 3. Axial post gadolinium T1 SE.

A progressive worsening with right hemidysmetria, ataxic gait, worsening of limb ataxia and dysarthria and dysphagia for solids, was observed for two following months. No weight loss, nausea or vomiting, headache or seizures were reported. There was no mention of fever and no respiratory, abdominal or genitourinary symptoms. The brain MRI was repeated and showed a growth of the expansive lesion, reason for which the patient was admitted to our Internal Medicine Department. 


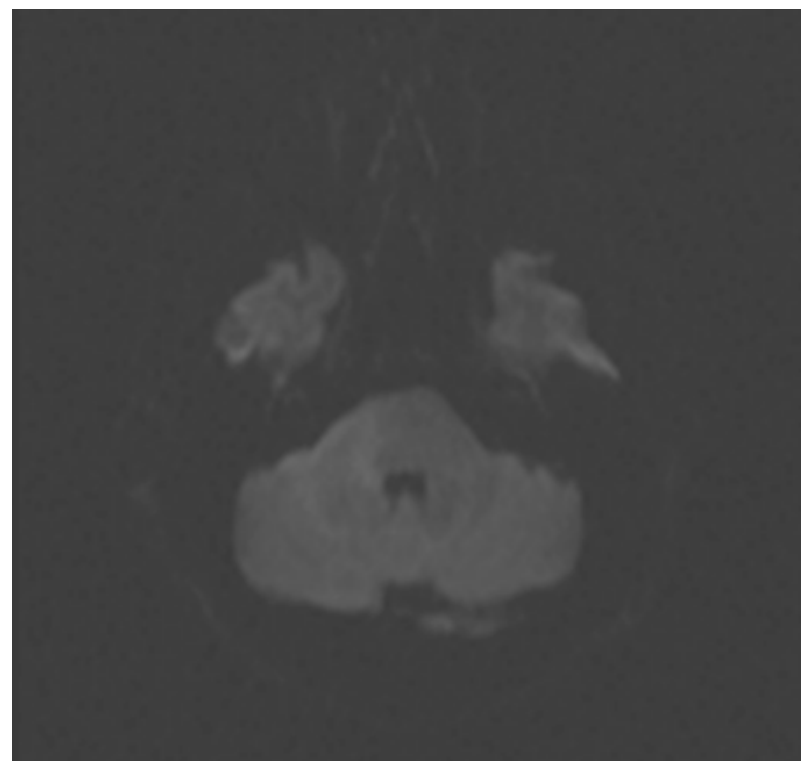

Figure 4. Axial DWI.

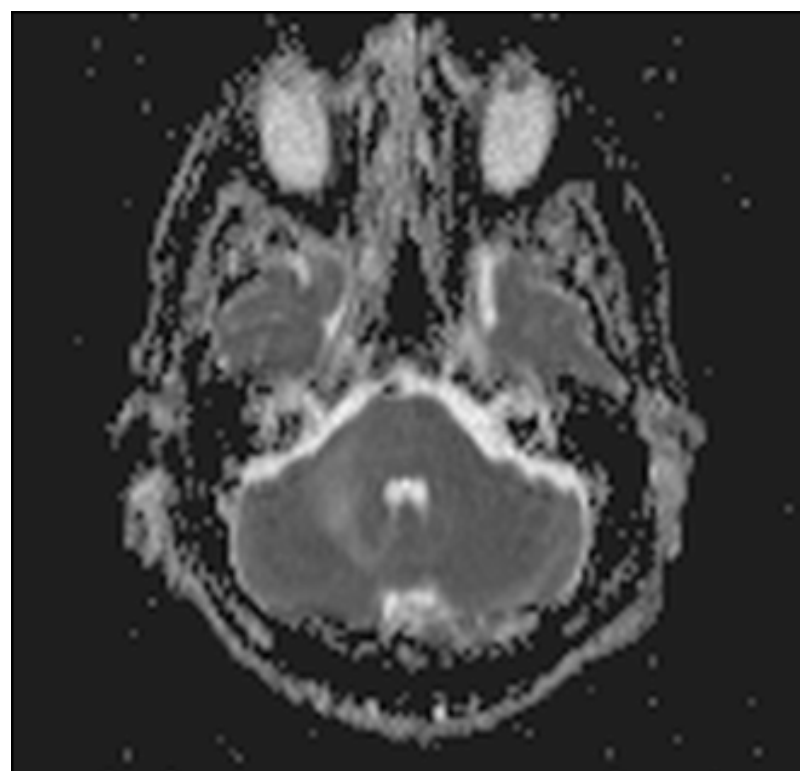

Figure 5. Axial MAPA ADC.

To what concerns the previous medical history, the patient had hypertension, dyslipidemia, degenerative osteoarticular disease and benign prostatic hyperplasia. The patient had no history of immunosuppressive therapies. The epidemiological context and family history were irrelevant. There was no consumption of tobacco, alcohol or illicit drugs and no sexual risk behaviour.

The examination on admission demonstrated that the blood pressure was $141 / 86 \mathrm{mmHg}$, the heart rate was 73 beats/minute (regular pulse) and the auricular temperature was $36.3^{\circ} \mathrm{C}$. The lung and heart auscultation showed no abnormalities and abdominal palpation revealed no organomegalies. No palpable adenomegalies and no skin or joint changes. The neurological examination 
showed the presence of dysarthria (without aphasia), right dysmetria in the finger-to-nose and heel-to-shin tests (without worsening when closing the eyes), dysdiadochokinesia in the rapid alternating movement test and ataxic gait in the heel toe walk test.

Laboratory exams revealed haemoglobin of $13.0 \mathrm{~g} / \mathrm{dL}$, leucocytes of $6.19 \times$ $10^{9} / \mathrm{L}$ (neutrophils of $4.18 \times 10^{9} / \mathrm{L}$ and lymphocytes of $1.28 \times 10^{9} / \mathrm{L}$ with 953 $\mathrm{CD} 4^{+}$), C-reactive protein of $12.5 \mathrm{mg} / \mathrm{L}$, a normal $\mathrm{LDH}$, and no hepatic or renal dysfunction. Serum levels of immunoglobulins and complement were normal. The peripheral blood immunophenotyping analysis revealed a polyclonality of $\mathrm{B}$ cells, with no changes in the T cells. During the course of hospitalization, transient lymphopenia was observed with a minimum lymphocyte count of $0.82 \times$ $10^{9} / \mathrm{L}$.

Taking into account the clinical course and particularly the imaging features, it was hypothesized that it could be a JC virus infection. HIV serology (HIV 1/2 screening with an antigen p24 plus antibodies test) was negative and HIV RNA was not detected. A lumbar puncture was performed which revealed total cells $2 / \mu \mathrm{L}$, proteins $0.85 \mathrm{~g} / \mathrm{L}$ and a normal $\mathrm{CSF} /$ serum glucose ratio. The JC virus DNA was positive in the CSF $\left(1.47 \times 10^{4}\right.$ copies $\left./ \mathrm{mL}\right)$ and in the blood $(<150$ copies $/ \mathrm{mL}$ ), determined by polymerase chain reaction (PCR). Accordingly, the definitive diagnosis of PML was assumed and treatment with mirtazapine was initiated. An obvious and progressive clinical and neurological worsening was observed and one month after the diagnosis the patient was aphasic and without the ability to have an autonomous gait. No further treatment was attempted, nor any further diagnostic or invasive intervention were performed. The death was occurred 1 year and 5 months after the diagnosis.

\section{Discussion}

PML occurs more frequently in immunocompromised patients and is a major opportunistic infection associated with HIV infection, making up approximately $80 \%$ of patients with PML. Other less common causes associated with PML are haematological malignancies (13\%), organ transplant recipients (5\%) and autoimmune diseases treated with immunomodulators (3\%) [3]. However, PML is no longer limited to the aforementioned groups, being described in patients with conditions associated with minimal immunosuppression, such as renal failure or liver cirrhosis. In addition, there are some reported cases of PML in patients without any apparent cause of immunosuppression [3]. After a review of the literature, we found about twenty-four cases described in individuals with no comorbidities and without known cause of immunosuppression, but we emphasize that in half of these cases the HIV serology was not reported.

The clinical manifestations of PML may be quite distinct, since any area of the brain may be virtually involved, taking into account the tropism of the JC virus to oligodendrocytes [2] [4]. PML is typically presented with subacute neurologic deficits, with the most common clinical findings being gait abnormalities, incoordination, motor weakness, visual field deficits, behavioural and cognitive ab- 
normalities, and language and speech disturbances [4]. PML is typically a bilateral, asymmetric and supratentorial white matter disease (typically parietal and frontal lobes), although it may be unilateral and with just a single lesion. The white matter of the posterior fossa is the next most commonly involved area, classically the middle cerebellar peduncles and adjacent pons and cerebellum, often synchronous with supratentorial lesions, being limited do infratentorial structures in about $10 \%$ of cases [2]. The MRI is the technique of choice, which reveals hyperintense lesions on T2-weighted and T2 FLAIR images and hypointense lesions on T1-weighted images in the involved brain regions, and peripheral patchy diffusion restriction may be present. Mass effect is infrequent and mostly minimum, and lesions may enhance in about $10 \%$ of cases, typically at the periphery. Although the brain biopsy is the gold standard for the diagnosis of PML, if the neurological and neuroradiological findings are suggestive, the diagnosis can be established by demonstrating the presence of JC virus DNA by PCR in the CSF [2] [4]. Thus, in this case the brain biopsy was not performed.

Although obvious immunosuppression exceedingly increases the risk of PML, many chronic diseases might be associated with a transient immune dysfunction which may promote the reactivation of the JC virus by impairing the ability to mount a $\mathrm{T}$ cell response. Another cause that has been associated with PML is idiopathic $\mathrm{CD}^{+} \mathrm{T}$-lymphocytopenia [5]. In this case, the initial neurological symptoms and the first brain MRI led to a misdiagnosis of a stroke. Subsequent clinical evolution and the imaging progression of the lesion raised other diagnostic hypotheses. Taking into account the extensive complementary study carried out, it was possible to exclude with some certainty that it was a neoplastic, lymphoproliferative, autoimmune disease. We emphasize that transient lymphopenia was observed during hospitalization (an immunophenotypic study was not performed at this time because this analytical change was only observed in a single study), and was not observed during subsequent analytical monitoring.

\section{Conclusions}

Associating the positive CSF JC virus PCR result to the highly suggestive clinical and imaging findings, it was possible to establish the diagnosis of PML. The cause of viral reactivation in our case remains unknown.

Currently, there is no specific antiviral agent for the JC virus infection and the existing treatment goal in PML is to restore host adaptive immune response (for example, antiretroviral therapy if HIV infection). Although the results were unsatisfactory, a number of specific drugs against the JC virus (as well as cidofovir, topotecan, cytarabine, mefloquine, mirtazapine) have been investigated. Mirtazapine has been implicated in preventing the progression of PML in some case reports [2] [5], which was not observed in our case.

\section{Conflicts of Interest}

The authors declare no conflicts of interest. 


\section{References}

[1] Tan, S.C. and Koralnik, I.J. (2015) JC, BK, and Other Polyomaviruses: Progressive Multifocal Leukoencephalopathy (PML). In: Bennett, J.E., Dolin, R. and Blaser, M.J., Eds., Mandell, Douglas, and Bennett's Principles and Practice of Infectious Diseases, 8th Edition, Elsevier Saunders, Philadelphia.

[2] Bag, A.K., Curé, J.K., Chapman, P.R., Roberson, G.H. and Shah, R. (2010) JC Virus Infection of the Brain. American Journal of Neuroradiology, 31, 1564-1576. https://doi.org/10.3174/ajnr.A2035

[3] Gheuens, S., Pierone, G., Peeters, P. and Koralnik, I.J. (2010) Progressive Multifocal Leukoencephalopathy in Individuals with Minimal or Occult Immunosuppression. Journal of Neurology, Neurosurgery \& Psychiatry, 81, 247-254. https://doi.org/10.1136/jnnp.2009.187666

[4] Berger, J.R., Aksamit, A.J., Clifford, D.B., Davis, L., Koralnik, I.J., Sejvar, J.J., et al. (2013) PML DIAGNOSTIC CRIteria: Consensus Statement from the AAN Neuroinfectious Disease Section. American Academy of Neurology, 80, 1430-1438. https://doi.org/10.1212/WNL.0b013e31828c2fa1

[5] Tan, C.S. and Koralnik, I.J. (2010) Progressive Multifocal Leukoencephalopathy and Other Disorders Caused by JC Virus: Clinical Features and Pathogenesis. Lancet Neurology, 9, 425-437. https://doi.org/10.1016/S1474-4422(10)70040-5 